\title{
Erratum to: Asymmetric polymers in bicontinuous microemulsions and their accretion to the bending of the membrane
}

\author{
Martin Brodeck • Simona Maccarrone • Debasish Saha • Lutz Willner • \\ Jürgen Allgaier • Gaetano Mangiapia • Henrich Frielinghaus • \\ Olaf Holderer • Antonio Faraone • Dieter Richter
}

Published online: 15 February 2015

(C) Springer-Verlag Berlin Heidelberg 2015

\section{Erratum to: Colloid Polym Sci}

\section{DOI 10.1007/s00396-014-3449}

The original version of this article, unfortunately contained errors.

The following is the list of errors and their corresponding correct presentation.

\begin{tabular}{|c|c|c|c|}
\hline Page number & Colum and Line number & Incorrect & Correct \\
\hline 3 & Col 2, line 22 & $\mathrm{FTP}=(T, \gamma)$ & $\mathrm{FTP}=(\widetilde{T}, \widetilde{\gamma})$ \\
\hline 3 & Col 2, line 39 & $(\propto \ln \Psi)$ & $(\propto \ln \widetilde{\psi})$ \\
\hline 3 & Col 2, line 41 & $\psi$ & $\widetilde{\psi}$ \\
\hline 3 & Col 2, line 44 & $\Xi=(6 \pi / 5) \bar{\Xi}$ & $\widehat{\Xi}=\left(\frac{6 \pi}{5}\right) \cdot \bar{\Xi}$ \\
\hline
\end{tabular}

The online version of the original article can be found at http://dx. doi.org/10.1007/s00396-014-3449-8.

\section{Brodeck}

Bonnenberg \& Drescher Projektentwicklung GmbH,

52457 Aldenhoven, Germany

S. Maccarrone $(\varangle) \cdot$ D. Saha $\cdot$ L. Willner $\cdot$ J. Allgaier $\cdot$

H. Frielinghaus $\cdot$ O. Holderer $\cdot$ D. Richter

Jülich Centre for Neutron Science JCNS, Forschungszentrum Jülich

$\mathrm{GmbH}$, Outstation at MLZ, Lichtenbergstr. 1, 85747 Garching,

Germany

e-mail: s.maccarrone@fz-juelich.de

D. Saha $\cdot$ L. Willner $\cdot$ J. Allgaier $\cdot$ D. Richter Forschungszentrum Jülich GmbH, Institute for Complex Systems ICS, 52425 Jülich, Germany
G. Mangiapia

G.S.A. Service, Via Edoardo Nicolardi 21, 80131 Naples, Italy

\section{A. Faraone}

NIST Center for Neutron Research, National Institute of Standards and Technology, 100 Bureau Drive, Gaithersburg, MD 20899-8562, USA

\footnotetext{
A. Faraone

Department of Materials Science and Engineering, University of Maryland, College Park, MD 20742, USA
} 


\begin{tabular}{|c|c|c|c|}
\hline Page number & Colum and Line number & Incorrect & Correct \\
\hline 4 & Col 1, line 8 & $C_{0, \mathrm{eff}} C_{0, \text { eff }}$ & $C_{0, e f f}$ \\
\hline 4 & Col 1, line 10 & $\widetilde{T} T$ & $\widetilde{T}$ \\
\hline 4 & $\mathrm{Col} 2$, line 3 & $\sigma R_{w / o} \sigma R_{W / O}$ & $\sigma R_{\mathrm{w} / \mathrm{o}}$ \\
\hline 4 & Col 2, line 13 & $\lambda=6.3 \AA(\Delta \lambda / \lambda=0.1) \lambda=6.3_{\AA}(\Delta \lambda / \lambda=0.1)$ & $\lambda=6.3 \AA(\Delta \lambda / \lambda=0.1)$ \\
\hline $\begin{array}{l}5 \\
5\end{array}$ & $\begin{array}{l}\text { Col 1, line } 6 \\
\text { Col } 2 \text {, line } 31\end{array}$ & $\begin{array}{l}\kappa_{\mathrm{R}, \mathrm{SANS}} \kappa_{R, S A N S} \\
\kappa / \kappa_{B} T \kappa / \kappa_{B} T\end{array}$ & $\begin{array}{l}\kappa_{R, S A N S} \\
\kappa / \kappa_{B} T\end{array}$ \\
\hline 5 & $\mathrm{Col} 2$, line 32 & $\kappa / \kappa_{B} T>>1 \kappa / \kappa_{B} T>>1$ & $\kappa / \kappa_{B} T \gg 1$ \\
\hline 5 & Col 2, line 33 & $\left(\sim \kappa^{-1 / 2} \kappa^{-1 / 2}\right)$ & $\left(\sim \kappa^{-1 / 2}\right)$ \\
\hline 6 & Col 1, line 11 & $T$ & $\widetilde{T}$ \\
\hline 6 & Col 1, line 22 & $\Xi=(4 \pi) / \bar{\alpha} 1 / 6$ & $\widehat{\Xi}=-(4 \pi) / \bar{\alpha} \cdot 1 / 6$ \\
\hline 6 & Col 1, lines $26,29,31,33,37$ & $\Xi$ & $\widehat{\Xi}$ \\
\hline 6 & Col 2, line 6 & $T$ & $\widetilde{T}$ \\
\hline 8 & Col 2, line 22 & $\Xi$ & $\widehat{\Xi}$ \\
\hline 10 & Col 1, line 24 & $T$ & $\widetilde{T}$ \\
\hline 10 & $14,21,23$ & $\Xi$ & $\widehat{\Xi}$ \\
\hline
\end{tabular}

In addition, the following shows the updated version of Scheme 2 image and caption.

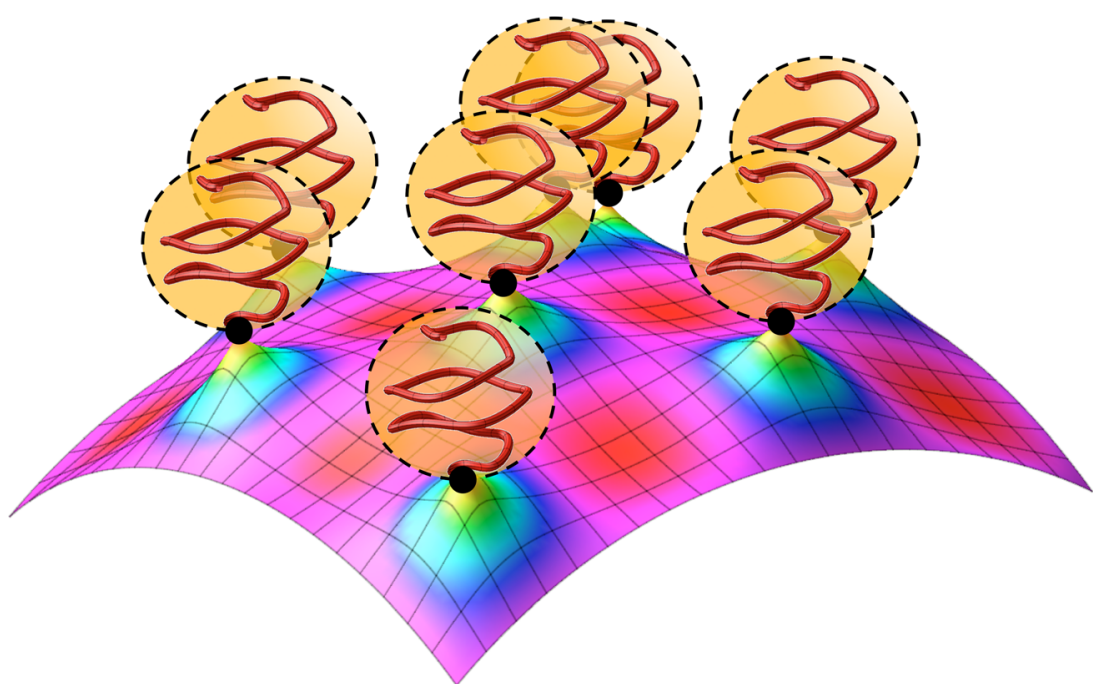

Scheme 2 Schematic representation of preferential accretion of the polymers to the curvature of the membrane that also shows small pinches at the anchoring points 